\title{
Failure of many ophthalmologists to use lasers safely
}

\author{
Kevin J Hardy, Jonathan R Lipton, David H Foster, John H B Scarpello
}

\begin{abstract}
In 1990, after the detection of impairment of colour discrimination in laser operators, the College of Ophthalmologists recommended safety guidelines for the use of lasers. We measured the effectiveness of these guidelines and their impact on ophthalmological practice in the United Kingdom. Previously, in ophthalmologists not following the guidelines, there was a deterioration in colour discrimination after a laser session. No such deterioration was found in 10 ophthalmologists tested who adhered to the guidelines, but their colour discrimination was significantly worse than that of controls. Replies to a questionnaire disclosed that one third of senior ophthalmologists were unaware of the practices recommended.
\end{abstract}

(Quality in Health Care 1992;1:168-170)

\section{Introduction}

The retina may be seriously damaged if excessive radiant energy is absorbed; short wavelength (blue) photons have higher energy and are thus potentially more harmful than longer wavelengths in this respect. ${ }^{1}$ The blue cones, which by definition absorb more of the shorter wavelengths of light, are thus particularly vulnerable to damage. ${ }^{2}$ It has been known for some time that diabetic patients may develop severe tritanopia (blue-green colour deficiency) after panretinal photocoagulation, ${ }^{3}$ but a potential hazard to clinicians using lasers has been appreciated only relatively recently. ${ }^{4}$ In 1989 Gunduz and Arden reported that colour contrast sensitivity, particularly at short wavelengths, was impaired in doctors using medical lasers. ${ }^{5}$ Subsequently they showed that the losses in colour discrimination were the result of "flashbacks" from the aiming beam hitting the surface of the contact lens used during laser treatment. ${ }^{6}$ As a result of this work they and the College of Ophthalmologists ${ }^{7}$ independently wrote to all ophthalmologists in the United Kingdom, recommending that argongreen lasers should be used in preference to argon-blue lasers, that appropriate short wavelength filters should be fitted to biomicroscopes, and that the intensity of the aiming beam used in photocoagulation treatment should be reduced.

We assessed the effectiveness of these guidelines and their impact on ophthalmological practice in the United Kingdom.

\section{Methods}

Using the Directory of Training Posts in Ophthalmology ${ }^{8}$ as a source, we sent a questionnaire to the consultant in charge of every NHS ophthalmology unit in the United Kingdom. The questionnaire assessed ophthalmologists' knowledge of laser induced damage to colour vision and their compliance with the College of Ophthalmologists' safety recommendations. Before its use in the survey the questionnaire was validated for a smaller number of ophthalmologists by means of a semistructured interview.

Using the Farnsworth-Munsell 100-hue test (100-hue test), ${ }^{9}$ a conventional clinical test, under standardised lighting conditions, we measured colour discrimination before and immediately after a laser session in 10 ophthalmologists (mean age 36(SD 10) years whose experience with lasers was between one and 10 years. All 10 ophthalmologists had normal Snellen acuity, and all had been following, for at least 12 months, safety guidelines to reduce the risk of laser induced loss of colour discrimination. Thus they all used an argon-green laser exclusively; they all used biomicroscopes fitted with appropriate short wavelength filters; and they all routinely reduced the intensity of the aiming beam during laser sessions. All 10 had previously used an argon-blue laser with a high intensity aiming beam and a biomicroscope without short wavelength filters. Colour discrimination in the ophthalmologists was compared with that in 10 healthy age matched controls. Statistical comparison between the ophthalmologists and controls was by Student's $t$ test and the Mann-Witney U test and comparison within ophthalmologists by a paired $t$ test.

\section{Results}

The response rate to the questionnaire was $55 \%(85 / 160)$. Each respondent (mean age 48 (7) years), was the consultant in charge of an ophthalmology unit had about $12(5)$ years of personal experience with lasers. Cumulative 
experience for the group was 984 years. Each consultant was responsible for five other users of lasers in his or her department (total 431 other users). Ninety eight per cent (157/160) of ophthalmology units used argon lasers. Most respondents (69/84 (82\%)) were aware that laser treatment could impair the colour vision of their patients, and 58/84 (70\%) were aware that it could adversely affect their own colour vision. Most (53/83 (64\%)) were aware that flashbacks were a potential cause of loss of colour discrimination in ophthalmologists, and $35 \%$ had experienced between one and 20 flashbacks during their career. One third of the consultants denied ever having been warned of the hazards for colour vision of laser treatment and precautions they might institute to avoid impairment of colour vision. A similar proportion did not reduce the intensity of the aiming beam, and many (38\%) did not know whether their biomicroscopes had been fitted with appropriate short wavelength filters. Only $27 \%(43 / 160)$ had had their colour vision assessed between 1985 and 1990 .

Colour discrimination in the 10 ophthalmologists we tested was significantly worse than that in the controls (mean (SE) 100-hue error score for ophthalmologists was 51.2(16.3) $v 14.8(4.8)$ for controls, $\mathrm{p}<0.01)$. Four of the ophthalmologists showed selective impairment of discrimination at short wavelengths compared with none of the controls. Colour discrimination in the ophthalmologists did not deteriorate after a laser session (100-hue error score before and after a laser session 51.2(16.3) $v$ 35.2(7.7)).

\section{Comment}

The survey encompassed most ophthalmology units in the United Kingdom. The respondents had a cumulative experience of lasers of 984 years and had 431 other users working in their departments. Despite publications in major ophthalmology journals in the United Kingdom and United States ${ }^{5} 6$ and written guidelines from the College of Ophthalmologists ${ }^{7}$ one third of senior ophthalmologists in the United Kingdom denied having received any communication about the potential hazard of laser treatment to their colour vision and were not taking appropriate precautions to protect their vision.

Although the number of subjects was small, we found, as previously, ${ }^{5610}$ that colour discrimination was significantly worse in ophthalmologists than in controls. Previous studies used a test of colour contrast sensitivity ${ }^{5610}$ whereas we used the Farnsworth-Munsell 100-hue test. ${ }^{9}$ We used a much brighter light source than is used traditionally, which may explain the greater sensitivity of the test in our hands. We found that colour discrimination did not deteriorate after a laser session, providing that the ophthalmologists followed the college's guidelines. This may reflect the effectiveness of the guidelines, although in subjects with established impairment of colour discrimination the 100-hue test may not be sufficiently sensitive to detect any further sight deterioration. If the acute deterioration in colour vision seen previously after laser use is a prelude to the more chronic impairment seen in ophthalmologists then the measures outlined by the college will probably prevent further visual damage among ophthalmologists. Losses in colour discrimination shown in this study and other studies would not be apparent to the subject, which may explain why some ophthalmologists have failed to adopt recommended safety practices; however, the long term sequelae of these subclinical colour deficits are unknown. The natural history of laser induced visual impairment is currently being evaluated by testing all ophthalmologists in the United Kingdom annually (G B Arden, personal communication). Until the results of this work are known every effort must be made to protect laser operators. Recommended safe practices seem to be effective, and they should be instituted as soon as is practicable in all ophthalmology units. In this respect, consultants in charge of ophthalmology units have a particular responsibility for the safety of colleagues working with lasers in their departments and should take steps to ensure the prompt adoption of safe practices.

The survey highlights a specific problem in ophthalmological practice in the United Kingdom, but laser induced loss of colour vision has also been shown in German ophthalmologists ${ }^{10}$ and is likely to be as widespread as the therapeutic use of lasers. It is not known what measures other countries have taken to protect those using medical lasers.

The survey also underlines several important general principles of audit. A problem has been identified (loss of colour vision among ophthalmologists), and a solution has been suggested (institution of the College of Ophthalmologists' safety guidelines). Audit has subsequently occurred at two levels: safety measures have been assessed and found to be effective, but their incorporation into clinical practice has been found to be deficient. There must now be a "closing of the feedback loop." " Efforts must be made to ensure that safety guidelines are incorporated into clinical practice, and then the audit process must be repeated.

We thank all the ophthalmologists who took part in this study and Professor G B Arden for his helpful advice. $\mathrm{KJH}$ is supported by a grant from Scotia Pharmaceutical, Guildford, Surrey.

1 Ham WT, Mueller HA, Ruffolo JJ, Guerry D, Guerry RK. Action spectrum for retinal injury from near-ultraviolet radiation in the aphakic monkey. Am $\mathfrak{f}$ Ophthalmol 1982;93:299-306.

2 Harwerth RS, Sperling HG. Prolonged color blindness induced by intense spectral lights in rhesus monkeys. Science $1971 \cdot 174 \cdot 520-3$.

3 Birch-Cox J. Defective colour vision in diabetic retinopathy before and after laser photocoagulation. Modern Problems of Ophthalmology 1978;19:326-9.

4 Sliney DH, Mainster MA. Potential laser hazards to the clinician during photocoagulation. $A m \boldsymbol{J}$ Ophthalmol clinician during phot

5 Gunduz $\mathrm{K}$, Arden GB. Changes in colour contrast sensitivity associated with operating argon lasers. $B r \mathcal{F}$ Ophthalmol 1989;73:241-6. 
6 Berninger TA, Canning CR, Gunduz K, Strong N, Arden GB. Using argon laser blue light reduces ophthalmologists' color contrast sensitivity. Arch Ophthalmol 1989;107:1453-8.

7 College of Ophthalmologists. Lasers. Quarterly Bulletin of the College of Ophthalmologists 1990:1.

8 Hawkins $\mathrm{R}$ ed. The directory of training posts in ophthalmologv: London: Hawker Publications, 1990.
9 Farnsworth D. The Farnsworth-Munsell 100-hue test manual.

10 Arden GB, Berninger T, Hogg CR, Perry S. A survey of color discrimination in German ophthalmologists. Changes associated with the use of lasers and operating microscopes. Ophthalmology 1991;98:567-71.

11 Smith T. Medical audit. BMF 1990;300:65.

列 